\title{
Proposta de um instrumento de medida para avaliar a satisfação de clientes de bancos utilizando a Teoria da Resposta ao Item
}

\author{
Proposal of tool to assess the satisfaction of bank \\ customers using the Item Response Theory
}

\author{
Alceu Balbim Junior ${ }^{1}$ \\ Antonio Cezar Bornia ${ }^{1}$
}

\begin{abstract}
Resumo: Este artigo apresenta um instrumento de medida para avaliação da satisfação de clientes de bancos utilizando a Teoria da Resposta ao Item (TRI). Satisfazer os clientes tem sido uma busca constante das organizações que procuram manterem-se competitivas no mercado. Estudos constatam a relação entre a qualidade percebida pelos clientes, a satisfação e fidelidade. A avaliação da satisfação pode ser realizada por meio da qualidade percebida pelos clientes e a construção de ferramentas de avaliação deve contemplar características específicas da atividade em questão. Embasando-se em artigos que avaliam a satisfação de clientes de bancos, propõe-se um instrumento formado por 29 itens. Os itens foram aplicados a 240 clientes a fim de avaliar a satisfação com o banco de maior relacionamento. Utilizando a Teoria da Resposta ao Item, foram identificados os parâmetros dos itens e a curva de informação. A análise do grau de discriminação dos itens indicou que todos são apropriados. A curva de informação obtida evidenciou o intervalo no qual o instrumento apresenta melhores estimativas para níveis de satisfação. O trabalho apresentou o nível médio de satisfação da amostra e a concentração de clientes nos diferentes níveis de satisfação da escala.
\end{abstract}

Palavras-chave: Satisfação de clientes. Bancos. Teoria da Resposta ao Item.

\begin{abstract}
This paper presents a model for assessing the satisfaction of bank customers using the Item Response Theory (IRT). Organizations are constantly making effort to satisfy customers seeking to remain competitive. Several studies have reported on the relationship between perceived quality, satisfaction, and loyalty. The assessment of satisfaction can be accomplished through the perceived quality, and the development of assessment tools should address specific features of the activity in question. Based on articles that assess the satisfaction of bank customers, this study proposes an assessment tool consisting of 29 items. The items were applied to 240 clients to assess their satisfaction with the bank with which they maintain a closer relationship. Using Item Response Theory (IRT), parameters of the items and the information curve of the tool proposed were identified. The analysis of the degree of discrimination of the items indicated that they all are appropriate. The information curve obtained showed the range in which the tool proposed produces the best estimates of satisfaction levels. The study revealed the average satisfaction level of the sample and the concentration of customers in different levels of satisfaction in the scale used.
\end{abstract}

Keywords: Customer satisfaction. Banks. Item Response Theory.

\section{Introdução}

Atuando em um ambiente de acirrada concorrência e de constantes instabilidades, as empresas buscam formas de se manterem competitivas. Neste contexto, a satisfação dos clientes torna-se um fator determinante na sobrevivência e conquista de espaço no mercado. A tecnologia que dinamiza o processo inovador atua sobre as informações e, com rapidez, concorrentes podem identificar e copiar iniciativas de sucesso. Mas, mesmo quando copiadas rapidamente, o período em que a empresa detém o domínio sobre a inovação pode, em caso de clientes pouco satisfeitos, influenciar a decisão de troca.

Este cenário é muito comum no setor bancário, no qual novos produtos são ofertados diariamente aos consumidores. Exemplos disso são os cartões de crédito sem custos de manutenção nem anuidades, os quais vêm se tornando item obrigatório no portfólio das instituições financeiras, assim como a oferta de serviços de pagamentos, transferências e informações via celular.

\footnotetext{
${ }^{1}$ Departamento de Engenharia de Produção e Sistemas, Universidade Federal de Santa Catarina - UFSC, CP 476, CEP 88040-900, Florianópolis, SC, e-mail: alceubalbim@yahoo.com.br; cezar@deps.ufsc.br
}

Recebido em 15/5/2010 - Aceito em 9/5/2011

Suporte financeiro: Nenhum. 
O foco das empresas, quando desenvolvem um novo produto ou copiam uma iniciativa de sucesso da concorrência, é elevar o nível da qualidade de suas ofertas, tornando-as mais adequadas às demandas de seus clientes, satisfazendo-os e tornando-os mais fiéis a sua marca.

Segundo a Federação Brasileira de Bancos (2009), em 2007 atuavam, no Brasil, 156 bancos que ofereciam seus produtos por meio de uma rede de atendimento com 147.857 dependências, divididos entre agências, postos tradicionais ou eletrônicos e correspondentes bancários. Já em 2010, segundo o Banco Central do Brasil (2010), o número de dependências totalizavam 152.848.

Desde 2007, todos os 5.659 municípios do País possuíam dependências bancárias - fruto de parcerias dos bancos com redes de supermercados, correios, padarias, lotéricas e outros estabelecimentos comerciais (FEDERAÇÃO..., 2009). Essas instituições comercializam seguros, previdência, aplicações, guarda de valores, cartões de crédito, investimento, empréstimos. Utilizam modernos recursos tecnológicos e têm no atendimento personalizado um aspecto de diferenciação. O setor bancário sofre regulação direta do Banco Central, que fiscaliza suas operações, disponibiliza um sistema de ouvidoria no seu site, avalia e divulga índices de reclamações e ranking de tarifas.

Os bancos elevam sua capacidade de atendimento e direcionam seus clientes para o canal de atendimento mais adequado. Focam seu trabalho em seguimentos específicos de clientes. Ao analisar o marketing bancário, Gosling e Gonçalves (2003) destacam que empresas apoiadas na tecnologia da informação conseguem ofertar produtos customizados a seus clientes e, como a maioria das empresas faz pagamentos de salários por meio de contas correntes, há interações constantes entre a empresa e o cliente e grandes oportunidades para o desenvolvimento de uma relação sólida e lucrativa.

Para manter elevada a qualidade percebida pelos clientes e atender a expectativas diversas, além de aumentar a rede de atendimento, os bancos necessitam investir constantemente na qualificação profissional e no desenvolvimento de produtos. O desafio dos gerentes é focar nas atividades que realmente atendam ou excedam as expectativas dos clientes. Para isso, é importante conhecer quais fatores são determinantes para os clientes, quando avaliam os serviços oferecidos pelos bancos (ZACHARIAS; FIGUEIREDO; ALMEIDA, 2008).

Considerando a necessidade de conhecer a satisfação dos clientes e os impactos da satisfação aos negócios, o objetivo deste artigo é elaborar um instrumento de medida para avaliar a satisfação dos clientes de bancos comerciais utilizando a Teoria da Resposta ao Item. Para isso, levantaram-se estudos que avaliam a satisfação dos clientes de bancos comerciais, obtendo-se as informações necessárias à elaboração do instrumento, o qual foi testado sob a ótica da TRI.

A TRI é uma metodologia estatística usada na medição de traços latentes (inteligência, percepção sobre determinado assunto, satisfação etc.), os quais não podem ser observados diretamente. Para determinar a magnitude de um traço latente em um indivíduo, a TRI gera uma escala, formada a partir de um conjunto de itens.

O artigo é composto por oito seções. A seção dois discorre sobre a satisfação dos clientes e a seção três apresenta e compara estudos que se propuseram a avaliar a satisfação de clientes de bancos. Na seção quatro, apresenta-se o instrumento de medida proposto, o qual foi criado a partir dos modelos analisados na seção anterior. Na seção cinco detalha-se a Teoria da Resposta ao Item e, na seguinte, a metodologia e a amostra. Por fim, a seção sete apresenta os resultados alcançados e na seção oito estão as conclusões.

\section{Análise da satisfação dos clientes}

Pode-se afirmar que a satisfação resulta da compreensão e tradução das necessidades dos clientes em características de produtos.

É o resultado alcançado quando as características dos produtos correspondem às necessidades dos clientes. Impactam as vendas, a participação no mercado e, consequentemente, os resultados financeiros (JURAN, 1997, p. 7).

A satisfação figura entre os principais objetivos gerenciais. De acordo com Paladini (2006, p. 142),

[...] os sistemas voltados para a gestão da qualidade total envolvem todos os requisitos para satisfazer as necessidades dos clientes, buscando sua fidelidade e acompanhando as variações do mercado.

Segundo Hansemark e Albinsson (2004) e Zeithaml, Berry e Parasuraman (1996), satisfação e retenção de clientes estão diretamente relacionadas a lucro e rentabilidade dos investimentos. Clientes satisfeitos e fiéis tendem a comprar mais, aumentar o nível de relacionamento com a empresa, pagar mais caro pelos produtos e fazer comentários positivos. Batenson e Hoffman (2001) destacam que a busca pela fidelização dos clientes é justificada porque os custos para manter um cliente são três a cinco vezes inferiores aos investimentos necessários para captar.

Para Hansemark e Albinsson (2004), a satisfação é uma atitude global do consumidor sobre um provedor de serviços, ou seja, a satisfação representa a atitude de realizar nova compra ou buscar outra empresa, de aumentar o nível de relacionamento ou manter o nível atual e avaliar melhor a empresa. Caruana (2002) 
desenvolveu uma pesquisa com base na relação entre qualidade, satisfação e fidelidade e identificou que a qualidade nos serviços antecede a satisfação e que a fidelidade tende a ser resultado tanto da satisfação quanto da qualidade. Olorunniwo, Hsu e Udo (2006) avaliaram a relação existente entre a qualidade nos serviços, satisfação, intenções e comportamento do consumidor (behavioral intentions). Concluíram que a correlação existente entre satisfação e o comportamento é forte e positiva, assim como a relação entre qualidade nos serviços e satisfação. Sureshchandar, Rajendram e Anantharaman (2002) aplicaram o mesmo questionário para avaliar a qualidade dos serviços e a satisfação dos clientes e identificaram que os clientes avaliam de forma diferente esses dois construtos. Concluíram que os construtos são diferentes, mas muito próximos e há relação estatística significante entre eles.

Ao conhecer o nível de satisfação dos clientes, tem-se um termômetro da capacidade de fidelizar clientes. Clientes fiéis compram mais, indicam novos clientes e fortalecem a marca que utilizam e em que confiam. Pode-se identificar o nível de satisfação pela percepção dos clientes sobre o produto.

No caso dos serviços, segurança e confiabilidade é o coração da excelência em serviços e a base para a diferenciação neste setor está relacionada aos seguintes fatores: i) definição clara do papel e da importância dos serviços de acordo com a perspectiva estratégica; ii) identificação, por meio de pesquisa, das expectativas dos clientes; iii) captação das informações sobre as estratégias dos concorrentes; iv) foco em fatores que impactam a satisfação dos clientes (poucos fatores); v) manutenção de serviços seguros e confiáveis; e vi) grande capacidade de identificar e solucionar problemas. (BERRY; ZEITHALM; PARASURAMAN, 1992, p. 225).

No ambiente de prestação de serviços, a gestão da qualidade centra-se fundamentalmente na interação com o usuário. Nesse processo interativo, destacam-se: i) a necessidade da gestão da qualidade priorizar o aspecto global, ou seja, todas as interações com o cliente; ii) a busca por ampliar o contato com o cliente; iii) a flexibilidade, criatividade e capacidade de adaptação às necessidades dos clientes; iv) a avaliação em todos os pontos de contato; e v) existência da coprodução (PALADINI, 2006, p. 193).

Os prestadores de serviços devem demonstrar segurança e confiabilidade em todos os contatos com o cliente. A empresa deve conhecer as expectativas, a percepção dos seus clientes, as estratégias dos concorrentes, focando no relacionamento e avaliando constantemente seus resultados.
Dois modelos se destacam na literatura com o objetivo de avaliar a qualidade dos serviços: o modelo apresentado por Parasuraman, Zeithaml e Berry (1988) - SERVQUAL - e o de Cronin e Taylor (1992) - SERVPERF. A diferença entre eles está basicamente na utilização das expectativas do consumidor na avaliação da satisfação. O SERVQUAL avalia a satisfação a partir da qualidade percebida e das expectativas com relação ao provedor de serviços. A SERVPERF utiliza apenas a qualidade percebida na avaliação da satisfação. Apesar destas diferenças, destaca-se a utilização de itens semelhantes na avaliação e opção dos autores por propor uma escala genérica, capaz de avaliar os diversos tipos de serviços.

Entretanto, a importância dos fatores-chave da satisfação varia de acordo com os grupos e os tipos de serviços (CHOWDHARY; PRAKASH, 2007). Essa afirmação remete à necessidade de incorporar elementos específicos do serviço avaliado.

Ladhari (2006) classifica as análises de satisfação em genéricas e específicas. As primeiras propõem itens para serem utilizadas na avaliação de qualquer setor ou segmento de serviços - como as escalas SERVQUAL e SERVPERF. As específicas são desenvolvidas para setores ou empresas. Para o autor, análises específicas são mais eficientes e podem derivar de modelos genéricos.

As propostas para avaliar a satisfação de clientes podem estar fundamentadas em características genéricas, ou focar elementos específicos de um determinado setor. A decisão implica considerar que uma escala genérica é capaz de oferecer todos os elementos necessários para tomada de decisão e alocação dos recursos necessários para elevar e manter a competitividade. Entretanto, Olorunniwo, Hsu e Udo (2006) afirmam que escalas específicas produzem resultados mais confiáveis.

Quanto mais aprofundado e detalhado for o estudo, melhor será o resultado. Chowdhary e Prakash (2007) desenvolvem um estudo com o objetivo de melhorar a compreensão desse tema. Sua pesquisa inclui hospitais, salões de beleza, academias, empresas de manutenção, lavanderia, companhias telefônicas, escolas, bancos, contabilidade, transporte e consultorias. O estudo procura identificar se características peculiares desses serviços impactam a avaliação e o comportamento dos clientes. Os autores verificam que os clientes são bastante consistentes, tanto nas análises quanto no ranking de importância dos atributos de qualidade de serviço. Grupos de clientes avaliam os critérios de forma diferente, assim como avaliam diferentes tipos de serviços por critérios diferentes. A importância dos fatores-chave varia de acordo com os grupos e os tipos de serviços.

Ladhari (2006) avalia trinta escalas de satisfação e identifica que os itens que apresentam melhores 
resultados variam de acordo com o país e o setor onde o modelo é aplicado. Ele verifica que, em alguns casos, a satisfação está relacionada a um construto unidimensional e, em outros, multidimensional. Completa afirmando que modelos genéricos não são suficientes, mas a adequação destes produz bons resultados. Para o autor, a maior contribuição do estudo está em reconhecer que cada contexto é único e deve ser considerado no desenvolvimento das escalas de satisfação, o que justifica a utilização de elementos e itens específicos.

\section{Mensuração da satisfação de clientes em bancos}

Johnston (1997) analisa a satisfação de clientes de bancos a partir da qualidade percebida e identifica a capacidade de satisfazer, de gerar insatisfação e a importância relativa de 18 variáveis relacionadas ao serviço bancário. As conclusões do estudo apontam maior relevância nas características intangíveis dos serviços - como o compromisso, atenção, amizade, cuidado e cortesia. Sugere que existem dois itens que permitem aos bancos obterem diferenciação: o compromisso e a atenção da equipe em todas as interações com o cliente.

Moutinho e Smith (2000) apontam relação direta entre a facilidade e agilidade de execução das transações, os relacionamentos interpessoais e o atendimento automático com a satisfação de clientes de bancos. Afirmam que os clientes procuram serviços ágeis e fáceis de executar. E que as estratégias dos bancos devem procurar, por meio do desenvolvimento tecnológico e humano, aprimorar constantemente seus serviços. Concluem que a agilidade dos serviços e o atendimento (humano e automatizado) impactam a capacidade de retenção (fidelidade).

Newman (2001) relata a utilização de uma escala SERVQUAL em um banco inglês e verifica que a aplicação contínua dessa ferramenta tem elevado os níveis de satisfação dos clientes, permitindo a identificação dos problemas e o direcionamento de ações para os setores responsáveis pelos resultados críticos.

Gounaris e Estathakopoulos (2001) avaliam a satisfação de clientes bancários na Grécia e concluem que os principais determinantes são a competência dos funcionários, a confiabilidade relativa à segurança nas transações, o ambiente, a aparência dos funcionários e equipamentos e as inovações nos produtos. Para os autores, devido à complexidade dos produtos, que podem envolver riscos de perda, os clientes preferem instituições que propiciem atendimento capaz de ajudar nas avaliações e contribuem para a segurança nas decisões.

Mihelis et al. (2001) afirmam que a satisfação dos clientes é dinâmica. Mudanças no mercado podem alterar suas preferências. Portanto, é necessário acompanhar os níveis de satisfação periodicamente, classificando os clientes em grupos, de acordo com a região de origem e características individuais e culturais comuns. Sua pesquisa identifica o acesso como categoria mais importante e com maior impacto na satisfação, seguido de pessoal, imagem, serviço e produtos.

Segundo Jamal e Naser (2002), a satisfação é um sentimento relacionado ao uso de produtos e serviços e pode definir o comportamento futuro dos clientes, tais como novas compras e propaganda a familiares e amigos. Detectam que é elevada a relação entre as dimensões essenciais, o relacionamento e a satisfação dos clientes. Nas dimensões essenciais, destacam-se pontualidade, precisão, qualidade e informação. Por fim, a expertise dos clientes impacta o nível de satisfação, assim como elementos culturais e geográficos.

Miguel (2002) avalia itens relacionados à infraestrutura, aos produtos, ao atendimento, à satisfação geral e aos determinantes da satisfação. Sua pesquisa solicita que os clientes informem os itens com maior capacidade de elevar a satisfação e os itens que impactam negativamente a satisfação. Identifica a qualidade no atendimento como o elemento com maior capacidade de elevar a satisfação e, como limitantes da satisfação, o tempo de espera, custos, localização e qualidade no atendimento. Destaca $12 \%$ dos clientes que consideram a qualidade dos equipamentos fator determinante da satisfação, $21 \%$ dos clientes que apontam o tempo como fator limitante da satisfação e $26 \%$ que consideram ruim ou regular o aceso à agência.

Horta (2002) argumenta que os bancos devem compreender as demandas da região em que atuam, adequando seu portfólio às necessidades dos clientes locais. Ele avalia a satisfação de médias empresas com o atendimento bancário e define os quatro elementos mais importantes para as empresas na relação comercial com os bancos: produtos, serviços, pessoas e instituição. Os elementos com maior impacto na satisfação são os produtos, a tecnologia e a capilaridade, relacionada ao tamanho dos bancos e suas áreas de abrangência.

Sureshchandar, Rajendran e Anhantharaman (2002) identificam correlação significativa entre qualidade e satisfação. Afirmam que a qualidade no atendimento tem maior influência na satisfação, seguida de sistemas, de tangíveis, de elementos essenciais e de responsabilidade social.

Patrício, Fisk e Cunha (2003) objetivaram avaliar fatores potenciais que influenciam a avaliação dos consumidores e o uso dos serviços via internet, acesso às agências, atendimento pelo telefone e terminais de autoatendimento. Os maiores benefícios dos canais de atendimento eletrônicos e telefônicos estão 
relacionados à acessibilidade, à conveniência e ao tempo. No caso das agências, esses itens tornam-se os pontos negativos. No entanto, as agências ganham quando se trata do atendimento personalizado, do apoio à decisão, da ajuda e do repasse de informações. Segundo o estudo, a maioria dos clientes faz uso de mais de um canal e pode-se imaginar que, de acordo com o tipo de serviço que desejam executar, recorrerão a diferentes canais.

Zhou (2004) por meio de análise fatorial exploratória dos itens da SERVQUAL gerou uma escala com três dimensões. Comparada às dimensões originais da SERVQUAL, a primeira agrega itens relacionados à empatia e à responsividade; a segunda agrega a confiabilidade e a segurança; e a terceira, os tangíveis. A proposta identifica relação direta entre a qualidade, a satisfação e as intenções do consumidor. As três dimensões apresentam confiabilidade elevada e os itens relacionados à dimensão empatia e à responsividade apresentam maior influência na satisfação.

Beerli, Martín e Quintana (2004) identificam que a relação entre satisfação, custos de mudança e fidelidade é positiva e estatisticamente significante, sendo que a satisfação tem maior influência sobre a fidelidade do que os custos de mudança. Segundo os autores, no setor bancário, o grau de sofisticação dos produtos exerce pequena influência nas decisões de troca.

Manrai e Manrai (2007) testam a relação existente entre a satisfação dos consumidores e a fidelidade. Concluem que os clientes percebem pouca diferença nos aspectos financeiros (como taxas e juros), mas veem grande diferença nos elementos não financeiros (como equipe e ambiente). Assim, aspectos não financeiros da atividade bancária exercem maior impacto na satisfação dos consumidores.

Vanniarajan e Anbazhagan (2007) utilizam a escala SERVPERF para avaliar a satisfação de clientes de bancos privados, públicos e mistos. Concluem que confiabilidade e segurança são os elementos com maior impacto na satisfação e que existe grande relação entre a qualidade percebida e a satisfação dos clientes. Com relação à confiabilidade, destacam-se a disposição para resolver problemas, informações corretas, empregados confiáveis e equipamentos modernos.

Em sua proposta, Zacharias, Figueiredo e Almeida (2008) consideram significativa a influência das seguintes variáveis na satisfação dos clientes: satisfação com a forma como o banco resolve problemas, satisfação com os gerentes do banco, satisfação com produtos e serviços do banco e satisfação com os caixas eletrônicos do banco.

O Quadro 1 resume os objetivos dos trabalhos, o número de itens e as conclusões sobre os principais fatores que definem a satisfação dos clientes.
As conclusões apontam para os componentes da atividade bancária com maior impacto na satisfação dos clientes. Destacam-se o atendimento e suas características (compromisso, atenção, amizade, cuidado, cortesia, agilidade, competência, informação, empatia, confiança e pontualidade) nos trabalhos de Zhou (2004), Sureshchandar, Rajendran e Anhantharaman (2002), Beerli et al. (2004), Jamal e Naser (2002), Moutinho e Smith (2000), Miguel (2002), Johnston (1997) e Gounaris e Stathakopoulos (2001).

Os produtos foram destaque nos trabalhos de Miguel (2002), Horta (2002), Gounaris e Stathakopoulos (2001), Mihelis et al. (2001), Beerli et al. (2004), e Zacharias, Figueiredo e Almeida (2008). Componentes dos produtos como taxas cobradas, rendimento recebido e inovações influenciam a satisfação dos clientes.

Os equipamentos e sistemas (tecnologia), sistemas de atendimento e acesso remoto e a aparência e modernidade dos equipamentos têm destaque nos resultados apresentados por Sureshchandar, Rajendran e Anhantharaman (2002), Zacharias, Figueiredo e Almeida (2008), Vanniarajan e Anbazhagan (2007), Beerli et al. (2004), Miguel (2002), Horta (2002) e Patrício, Fisk e Cunha (2003).

$\mathrm{O}$ acesso e itens correspondentes como acessibilidade, conveniência, facilidade, estacionamento e capilaridade são destaque nos resultados de Moutinho e Smith (2000), Miguel (2002), Horta (2002), Mihelis et al. (2001) e Patrício, Fisk e Cunha (2003).

Com relação à composição das análises, tem-se que os números de itens variam entre 7 e 35 . Quanto aos objetivos, Johnston (1997) e Horta (2002), Miguel (2001) e Moutinho e Smith (2000) além de avaliar a satisfação, buscam mensurar a importância relativa dos itens. E Manrai e Manrai (2004), Beerli, Martín e Quintana (2004), Jamal e Naser (2002), Sureshchandar, Rajendran e Anantharaman (2002) e Zhou (2004) buscam identificar também a relação existente entre qualidade, fidelidade e satisfação.

Dentre os principais fatores que influenciam a satisfação dos clientes, destacam-se o atendimento, presente em onze trabalhos, os equipamentos e sistemas (sete trabalhos), os produtos com (seis citações) e o acesso aos serviços e instalações, sejam de forma física ou eletrônica (cinco).

Com relação aos itens utilizados na avaliação do atendimento, destacam-se a aparência dos funcionários (oito citações), informações prestadas (sete citações), confiabilidade e disponibilidade (cinco citações) e competência (quatro citações). Para avaliar equipamentos e sistemas, são utilizados itens relacionados aos equipamentos (quatro citações), modernidade, terminais de atendimento e sistemas (duas citações). Para avaliar os produtos, destacam-se 
Quadro 1. Itens e dimensões utilizadas na avaliação da satisfação de clientes de bancos comerciais.

\begin{tabular}{|c|c|c|c|c|}
\hline Autores & Ano & Objetivos & $\begin{array}{l}N^{0} \text { de } \\
\text { itens }\end{array}$ & $\begin{array}{c}\text { Fatores principais na } \\
\text { satisfação - resultados }\end{array}$ \\
\hline Johnston & 1997 & $\begin{array}{l}\text { Identificar capacidade de } \\
\text { satisfazer, gerar insatisfação e } \\
\text { importância relativa dos itens. }\end{array}$ & 18 & Compromisso e atenção. \\
\hline Moutinho e Smith & 2000 & $\begin{array}{l}\text { Identificar os fatores que } \\
\text { contribuem para a } \\
\text { satisfação e fidelidade. }\end{array}$ & 19 & $\begin{array}{l}\text { Serviços ágeis, fáceis de } \\
\text { executar e equipe treinada. }\end{array}$ \\
\hline Newman & 2001 & $\begin{array}{l}\text { Avaliar resultados da } \\
\text { aplicação SERVQUAL } \\
\text { em um banco comercial. }\end{array}$ & 19 & $\begin{array}{l}\text { Escala permite o acompanhamento } \\
\text { sistemático do nível de satisfação e a } \\
\text { identificação das atividades que não } \\
\text { contribuem para a satisfação dos clientes. }\end{array}$ \\
\hline $\begin{array}{l}\text { Gounaris e } \\
\text { Estathakopoulos }\end{array}$ & 2001 & $\begin{array}{l}\text { Avaliar a satisfação } \\
\text { dos clientes bancários. }\end{array}$ & 25 & $\begin{array}{c}\text { Confiabilidade, seguida da competência } \\
\text { dos empregados, aparência e } \\
\text { rentabilidade. }\end{array}$ \\
\hline Mihelis et al. & 2001 & $\begin{array}{l}\text { Avaliar a satisfação } \\
\text { dos clientes bancários. }\end{array}$ & 18 & $\begin{array}{l}\text { Em ordem de importância acesso, } \\
\text { pessoal, imagem, serviços e produtos. }\end{array}$ \\
\hline Jamal e Naser & 2002 & $\begin{array}{l}\text { Relacionar a qualidade, } \\
\text { expectativas e características } \\
\text { individuais com a satisfação. }\end{array}$ & 20 & $\begin{array}{l}\text { A pontualidade das entregas e do serviço, } \\
\text { a qualidade constante do atendimento, } \\
\text { a precisão, o nível de informações } \\
\text { prestadas, a disposição, a cortesia e a } \\
\text { capacidade de atendimento. }\end{array}$ \\
\hline Miguel & 2002 & $\begin{array}{c}\text { Identificar o nível de } \\
\text { satisfação e os fatores } \\
\text { determinantes de satisfação. }\end{array}$ & 17 & $\begin{array}{l}\text { Qualidade no atendimento; infraestrutura } \\
\text { (localização, a comodidade, a } \\
\text { modernidade e o estacionamento); } \\
\text { produtos (facilidade de obter } \\
\text { empréstimos e taxas cobradas) e as } \\
\text { transações por internet e telefone. }\end{array}$ \\
\hline Sureshchander et al. & 2002 & $\begin{array}{l}\text { Identificar se qualidade e } \\
\text { satisfação são construtos distintos } \\
\text { e se possuem correlação. }\end{array}$ & 39 & $\begin{array}{l}\text { A qualidade no atendimento, seguida de } \\
\text { sistemas, tangíveis, elementos essenciais } \\
\text { e responsabilidade social. }\end{array}$ \\
\hline Gosling e Gonçalves & 2003 & $\begin{array}{l}\text { Validar escala para avaliar a } \\
\text { satisfação de clientes. }\end{array}$ & 36 & $\begin{array}{l}\text { Dos } 7 \text { elementos, apenas propensão } \\
\text { a deixar o relacionamento e conflito } \\
\text { funcional não foram validados. }\end{array}$ \\
\hline Horta & 2003 & $\begin{array}{c}\text { Avaliar a satisfação e determinar } \\
\text { os elementos mais importantes } \\
\text { para os clientes. }\end{array}$ & 35 & $\begin{array}{c}\text { O portfólio de produtos, as taxas } \\
\text { cobradas, disponibilidade de crédito, } \\
\text { tecnologia e capilaridade. }\end{array}$ \\
\hline Patrício et al. & 2003 & $\begin{array}{l}\text { Identificar fatores que impactam } \\
\text { a utilização de caixas eletrônicos } \\
\text { e outros canais de atendimento. }\end{array}$ & & $\begin{array}{c}\text { A acessibilidade, a conveniência, } \\
\text { o tempo despendido, o atendimento } \\
\text { personalizado, o apoio à decisão e o } \\
\text { nível de informação. }\end{array}$ \\
\hline Zhou & 2004 & $\begin{array}{l}\text { Avaliar a relação entre qualidade, } \\
\text { satisfação e as intenções e } \\
\text { comportamentos do consumidor. }\end{array}$ & 21 & $\begin{array}{l}\text { Personalização do atendimento, } \\
\text { compreensão das necessidades, } \\
\text { atenção, disposição, nível de erros e } \\
\text { conhecimento. }\end{array}$ \\
\hline Beerli et al. & 2004 & $\begin{array}{l}\text { Identificar os antecedentes da } \\
\text { satisfação, qualidade } \\
\text { percebida e fidelidade. }\end{array}$ & 33 & $\begin{array}{l}\text { O nível de sofisticação dos } \\
\text { produtos exerce baixa influência } \\
\text { na satisfação e modernidade dos } \\
\text { equipamentos e o atendimento têm forte } \\
\text { peso na satisfação global. }\end{array}$ \\
\hline $\begin{array}{l}\text { Vanniarajan e } \\
\text { Anbazhagan }\end{array}$ & 2007 & $\begin{array}{c}\text { Avaliar a satisfação } \\
\text { dos clientes bancários. }\end{array}$ & 17 & $\begin{array}{l}\text { Confiabilidade, segurança, disposição } \\
\text { para resolver problemas, informações } \\
\text { corretas, empregados confiáveis e } \\
\text { equipamentos modernos. }\end{array}$ \\
\hline Manrai e Manrai & 2007 & $\begin{array}{l}\text { Testar relação entre a satisfação } \\
\text { e a probabilidade de mudar de } \\
\text { instituição. }\end{array}$ & 24 & $\begin{array}{l}\text { Satisfação tem forte influência na } \\
\text { probabilidade de mudar de banco. } \\
\text { Aspectos humanos têm maior influência } \\
\text { na satisfação que aspectos financeiros. }\end{array}$ \\
\hline Zacharias et al. & 2008 & $\begin{array}{l}\text { Avaliar a relação existente entre } \\
\text { a satisfação, os produtos e os } \\
\text { serviços, os equipamentos, o } \\
\text { atendimento e a capacidade de } \\
\text { resolver problemas. }\end{array}$ & 7 & $\begin{array}{l}\text { Capacidade de resolver problemas, } \\
\text { atendimento dos gerentes, produtos e } \\
\text { serviços e caixas eletrônicos. }\end{array}$ \\
\hline
\end{tabular}


taxas e custos (sete citações) e portfólio (cinco citações). No acesso, tem-se a segurança (seis citações), localização e facilidade (cinco citações) e organização (três citações).

\section{Instrumento de medida proposto para avaliar a satisfação de clientes de bancos comerciais}

A fim de elaborar um instrumento para avaliar a satisfação de clientes de bancos, observaram-se os fatores e itens citados em mais pesquisas, conforme apresentados na seção anterior. Além disto, buscou-se agregar um conjunto de itens de forma a representar as características da atividade bancária.

Dessa forma, o instrumento proposto considerou nas características dos produtos itens relacionados à funcionalidade, aos custos, aos benefícios e às taxas de juros. Na avaliação do atendimento, consideraram-se a cortesia, a atenção, a disponibilidade, a competência e a confiabilidade. Nos equipamentos e sistemas, os itens investigam se os clientes encontram-se satisfeitos com os equipamentos e sistemas utilizados no atendimento. Com relação ao acesso, avalia-se o acesso via internet, os terminais de autoatendimento, as agências, a segurança nesses ambientes e a localização.

Desta maneira foi estruturado um conjunto de itens agrupados em 4 grupos: atendimento, produtos, acesso e equipamentos e sistemas. O Quadro 2 apresenta esses grupos e os itens que ele procura englobar.

Quatro itens utilizados que avaliam o atendimento têm origem direta nos modelos apresentados e buscam avaliar: aparência dos funcionários, competência, cortesia e confiabilidade e clareza das informações. Outros sete itens completam a avaliação e buscam agrupar as características principais do atendimento. Ao avaliar o tempo que os clientes gastam para realizar suas transações, busca-se compreender agilidade, atenção, eficiência, interesse, habilidade, ajuda e o desempenho da equipe de atendimento. No cumprimento dos horários e prazos, busca-se identificar a pontualidade, compromisso e credibilidade. $\mathrm{Na}$ competência no atendimento, capta-se a percepção sobre a qualificação, pois apenas pessoas qualificadas para executar uma função conseguem passar a imagem de competente, e, na avaliação da cortesia, capta-se o cuidado e a empatia.

A tecnologia está presente na maioria das atividades bancárias. Na avaliação dos equipamentos e sistemas, os clientes são capazes de identificar o nível de modernidade e a tecnologia utilizada pelo banco. A qualidade dos sistemas avalia a percepção dos clientes quando utilizam sistemas para pagamento e gerenciamento da conta.

A avaliação dos produtos compõem-se de oito itens relacionados a: rentabilidade, custos dos produtos, disponibilidade de crédito e adequação dos produtos às necessidades dos clientes. O portfólio é avaliado pela variedade de produtos. A utilidade está relacionada aos benefícios obtidos com a aquisição, e a flexibilidade está relacionada aos benefícios, à adequação e à disponibilidade.

O último grupo de itens está relacionado ao acesso. A satisfação com o acesso foca-se nas condições que a empresa fornece para seus clientes. Ele engloba a segurança como fator muito importante nos acessos dos clientes, por meio de três itens: segurança nos acessos às agências, nos terminais eletrônicos e na internet. Dois itens avaliam a infraestrutura da agência e englobam o espaço físico, layout, conforto, estética, estacionamento e organização.

Os serviços de internet e a home page são avaliados pela segurança e pelo acesso à internet. A avaliação do horário está relacionada ao período de atendimento nas agências e nos terminais de autoatendimento. $\mathrm{O}$ Quadro 3 apresenta o instrumento de medida utilizado. Portanto, para avaliação da satisfação dos clientes de bancos, propõem-se 29 itens, sendo 11 itens relacionados ao atendimento, oito relacionados aos produtos, 2 relacionados a equipamentos e sistemas e 8 ao acesso. Sugere-se que todos os itens sejam observados em uma escala ordinal de 5 níveis.

\section{A Teoria da Resposta ao Item}

Os modelos baseados na Teoria da Reposta ao Item definem o nível do traço latente avaliado (habilidade, satisfação, conformidade) por meio da probabilidade do indivíduo dar determinada resposta aos itens de um questionário. No caso da satisfação dos clientes bancários, quanto maior a satisfação, maior será a

Quadro 2. Determinantes da satisfação de clientes bancários.

\begin{tabular}{|lr|}
\hline Elementos da qualidade & Principais determinantes da satisfação \\
\hline Atendimento & Conhecimento; Compromisso; Atenção; Agilidade; Confiabilidade; \\
& Disposição; Informação; Competência; Aparência; Pontualidade; \\
Precisão; Cortesia; Capacidade; Apoio à Decisão; Compreensão; Erros. \\
Equipamentos e sistemas & Modernidade; Caixas Eletrônicos; Sistemas; Outros Equipamentos. \\
Produtos & Facilidade Obter Empréstimos; Taxas Cobradas; Portfólio; Rentabilidade. \\
Acesso & Localização; Estacionamento; Internet; \\
& Segurança; Conveniência; Tempo Gasto; Comodidade. \\
\hline
\end{tabular}


Quadro 3. Instrumento de medida proposto.

\begin{tabular}{|c|c|}
\hline & Instrumento de medida proposto para avaliar a satisfação \\
\hline \multicolumn{2}{|c|}{$\begin{array}{l}\text { Avalie o atendimento (agilidade, competência, cortesia e aparências dos funcionários da instituição), } \\
\text { considerando os seguintes itens: }\end{array}$} \\
\hline ATEN1 & 1. Aparência dos funcionários \\
\hline ATEN2 & 2. Competência dos funcionários para resolver seus problemas... \\
\hline ATEN3 & 3. Cortesia no atendimento - no telefone e pessoalmente... \\
\hline ATEN4 & 4. Tempo gasto para resolver problemas na Agência... \\
\hline ATEN5 & 5. Tempo gasto para resolver problemas nos terminais de autoatendimento... \\
\hline ATEN6 & 6. Tempo gasto para resolver problemas via Internet... \\
\hline ATEN7 & 7. Tempo que os funcionários levam para responder suas dúvidas... \\
\hline ATEN8 & 8. Tempo de resposta às suas reclamações... \\
\hline ATEN9 & 9. Tempo de resposta e atendimento de suas solicitações/demandas... \\
\hline ATEN10 & 10. Cumprimento dos horários e prazos... \\
\hline ATEN11 & 11. Confiabilidade e clareza das informações... \\
\hline \multicolumn{2}{|c|}{$\begin{array}{l}\text { Avalie os produtos do banco que você utiliza (seguros, investimentos, adiantamentos, empréstimos, contas e } \\
\text { outros), considerando os seguintes itens: }\end{array}$} \\
\hline PROD1 & 1. Rentabilidade (juros recebidos)... \\
\hline PROD2 & 2. Custo dos produtos... \\
\hline PROD3 & 3. Variedade de produtos e serviços... \\
\hline PROD4 & 4. Adequação dos produtos às suas necessidades... \\
\hline PROD5 & 5. Disponibilidade de crédito... \\
\hline PROD6 & 6. Agilidade na concessão do crédito... \\
\hline PROD7 & 7. Burocracia na concessão de crédito e contratação de produtos... \\
\hline PROD8 & 8. Benefícios obtidos com os produtos... \\
\hline \multicolumn{2}{|c|}{$\begin{array}{l}\text { Avalie os equipamentos (computadores das agências e terminais de autoatendimento) e os sistemas, } \\
\text { considerando os seguintes itens: }\end{array}$} \\
\hline EQPA1 & 1. Aparência dos equipamentos utilizados no atendimento... \\
\hline EQPA2 & 2. Sistemas que você tem acesso... \\
\hline \multicolumn{2}{|c|}{ Avalie a facilidade de acesso aos produtos do banco, considerando os seguintes itens: } \\
\hline ACES1 & 1. Horário disponível para atendimento na agência... \\
\hline ACES2 & 2. Horário de funcionamento dos terminais de autoatendimento... \\
\hline ACES3 & 3. Infraestrutura da agência (espaço físico, mobília e organização)... \\
\hline ACES4 & 4. Infraestrutura da agência (estacionamento)... \\
\hline ACES5 & 5. Acesso disponível via internet... \\
\hline ACES6 & 6. Segurança quando acessa seu banco via terminais de autoatendimento... \\
\hline ACES7 & 7. Segurança quando acessa seu banco nas agências... \\
\hline ACES8 & 8. Segurança quando acessa seu banco via internet... \\
\hline
\end{tabular}

probabilidade de o cliente atribuir níveis mais altos ao item avaliado, considerando uma escala ordinal. Em uma escala dicotômica, maior será a probabilidade de resposta positiva.

Atualmente, em várias áreas do conhecimento, particularmente em avaliação educacional, vem crescendo o interesse na aplicação de técnicas derivadas da Teoria de Resposta ao Item - TRI, que propõe modelos para os traços latentes, ou seja, características do indivíduo que não podem ser observadas diretamente. Esse tipo de variável deve ser inferido a partir da observação de variáveis secundárias que estejam relacionadas a ela. O que esta metodologia sugere são formas de representar 
a relação entre a probabilidade de um indivíduo dar certa resposta a um item e seus traços latentes, proficiências ou habilidades na área de conhecimento avaliada (ANDRADE; TAVARES; VALLE, 2000).

A TRI fornece com precisão a quantidade de informação de um item na escala de análise do traço latente e da escala. Em uma escala de satisfação, pode-se avaliar qual nível de satisfação que o item avalia e o volume de informação que oferece.

Em resumo, Reise, Ainsworth e Haviland (2005), Primi (2004), Nunes e Primi (2005), Alexandre et al. (2003) e Andrade, Tavares e Valle (2000) afirmam que a TRI é um conjunto de modelos matemáticos e estatísticos que formaliza a relação entre a probabilidade de responder corretamente ou positivamente um conjunto de itens e a habilidade (traço latente) necessário para tal. É possível construir uma função que expresse a relação entre a probabilidade de responder positivamente e a satisfação, quanto mais elevado o nível do traço latente maior a probabilidade da resposta ser positiva.

Os paramentos dos itens propostos para avaliar a satisfação dos clientes de bancos comerciais e o nível de satisfação destes clientes serão mensurados a partir do modelo logístico unidimensional para itens dicotômicos com dois parâmetros, apresentado na Equação 1.

$$
P\left(U_{i j}=1 \mid \theta_{j}\right)=\frac{1}{1+e^{-a_{i}}\left(\theta_{j}-b_{i}\right)}
$$

Na Equação 1, $\mathrm{U}_{\mathrm{ij}}$ representa a variável dicotômica que assume os valores 1 - para respostas positivas - ou 0 - quando o indivíduo $j$ não responde positivamente ao item $i$. O parâmetro $\theta_{\mathrm{j}}$ representa a satisfação (traço latente) do j-ésimo indivíduo. $\mathrm{P}\left(\mathrm{U}_{\mathrm{ij}}=1 \mid \theta_{\mathrm{j}}\right)$ é a probabilidade de um indivíduo $j$ com satisfação $\theta \mathrm{j}$ responder positivamente o item $i$ e é chamada de Função de Resposta do Item - FRI. O parâmetro $a_{i}$ é o parâmetro de discriminação (ou de inclinação) do item $i$, com valor proporcional à inclinação da Curva Característica do Item - CCI no ponto $b_{i}$. Quanto maior o seu valor, maior será a inclinação e a possibilidade de discriminação do item na escala de satisfação, ou seja, o item terá maior capacidade de diferenciar pessoas com níveis distintos no construto analisado. O parâmetro $b_{i}$ é o parâmetro de dificuldade (ou de posição) do item $i$, medido na mesma escala da habilidade. Ele representa a satisfação necessária para uma probabilidade de resposta positiva igual $0,5(50 \%)$. Pode-se afirmar que o valor de $b$ é o ponto em que a curva característica do item tem sua maior inclinação.

Assim como pela qualidade dos parâmetros $a$ e $b$, a validade de um item pela TRI está relacionada à quantidade de informação nele contida. Essa característica é identificada pela curva de informação. Conforme Baker (2001), Soares (2005), Uttaro e
Lehmann (1999), Reise, Ainsworth e Haviland (2005), a função de informação do item deriva da função de informação de Fisher. Define a colaboração do item para a precisão com que as habilidades são estimadas. Segundo Andrade, Tavares e Valle (2000), a função de informação no modelo logístico de três parâmetros pode ser escrita como (Equação 2):

$$
l_{i}(\theta)=a_{i}^{2} Q_{i}(\theta) \mathrm{P}_{\mathrm{i}}(\theta)
$$

Segundo Andrade, Tavares e Valle (2000), $\mathrm{I}_{\mathrm{i}}(\theta)$ representa a quantidade de informação do item $i$ no nível de satisfação $\theta$ e há mais informação em um item quando $b_{i}$ se aproxima de $\theta$ e quanto maior for $o a_{i}$. As curvas de informação do item permitem identificar a área da escala em que o item é mais preciso. Diferentes itens geram diferentes graus de informação sobre um traço latente (construto), sendo os itens relacionados a um menor nível da escala, mais úteis para diferenciar pessoas com baixa satisfação. A Figura 1 apresenta as curvas de informação de 3 itens hipotéticos.

As soma das informações dos itens resulta na informação do teste. A curva de informação do teste, por sua vez, representa a informação contida no conjunto de itens para cada nível da escala. Elevados níveis de informação ou confiabilidade tornam as estimativas do construto satisfação $(\theta)$ mais próximas dos escores observados (UTTARO; LEHMANN, 1999), ou seja, mede-se melhor a relação entre a variação no traço latente e os resultados da escala de pesquisa.

Quanto mais informação, melhor será a escala - quanto maior o volume de informação na escala, mais precisa será a medida (REISE; AINSWORTH; HAVILAND, 2005, p. 96, tradução nossa).

A validade dos itens e da ferramenta proposta está relacionada a sua capacidade de avaliar a satisfação dos clientes a partir de um conjunto de itens que

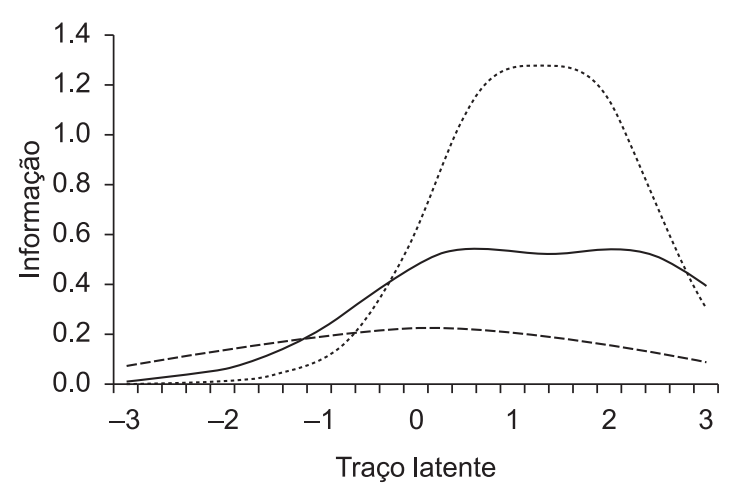

Figura 1. Curva de informação de três itens. Fonte: Uttaro e Lehmann (1999). 
caracterizam a atividade bancária. Essa adequação é representada pela curva de informação e capacidade de discriminação gerada pelo parâmetro $a$ dos itens.

\section{Metodologia, amostra e análise de dados}

Quatro etapas compuseram o desenvolvimento deste trabalho. Na primeira, fez-se a identificação do conjunto de itens, por meio de pesquisa bibliográfica. Os itens propostos foram avaliados por um especialista em TRI, e, antes de serem aplicados, os itens foram encaminhados para verificação de aplicabilidade, na qual dez clientes avaliaram a clareza das questões e o conteúdo. As fases finais compreendem a aplicação do instrumento e a análise dos dados e resultados (Figura 2).

A técnica utilizada foi o levantamento por meio de questionário. Do ponto de vista da sua natureza, essa pesquisa é aplicada. Utiliza as técnicas matemáticas e estatísticas da TRI em uma abordagem quantitativa. Como envolve a descrição de características de uma população específica pela aplicação de um questionário, estabelecendo relações entre as variáveis observadas e a satisfação dos clientes bancários, a pesquisa classifica-se, conforme Gil (1991), como descritiva.

A pesquisa foi realizada com empresários e gerentes financeiros de empresas situadas nos municípios de Florianópolis e São José em Santa Catarina. Quando houve respondentes com contas em mais de um banco, foram solicitadas informações a respeito do banco com o qual mantinham maior relacionamento.

Os questionários foram entregues diretamente aos responsáveis para preenchimento e recolhidos após alguns dias. Somaram-se aos questionários recolhidos nas empresas quatro questionários respondidos via internet. Ao total foram utilizadas 240 respostas. A análise dos dados foi realizada com o software BILOG-MG, desenvolvido por Mislevy D. J. e Bock R. D. Devido ao reduzido tamanho da amostra,

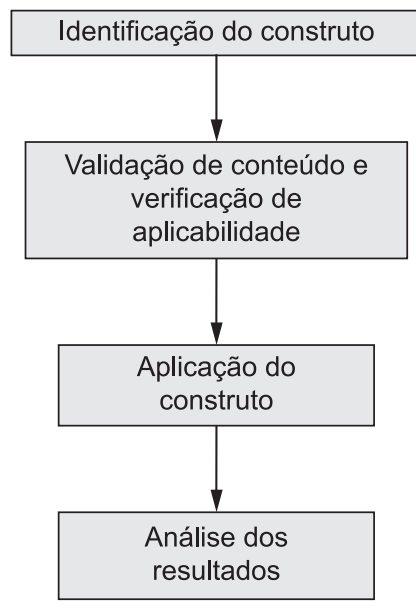

Figura 2. Fases da pesquisa. optou-se por dicotomizar as respostas e utilizar o modelo logístico de 2 parâmetros. Desta forma, clientes que indicaram nível de satisfação entre 1 e 3 foram reclassificados como insatisfeitos e clientes com nível de satisfação 4 ou 5 foram reclassificados como satisfeitos.

\section{Resultados}

Os resultados da aplicação do conjunto de itens são apresentados na Tabela 1. Ela apresenta os itens, a quantidade de respostas obtidas para cada item e as estimativas dos parâmetros $a$ e $b$, com seus respectivos erros padrões.

O processo de calibração define os parâmetros dos itens. Nestes resultados identifica-se o nível de discriminação de um item, parâmetro $a$, e o nível de satisfação relacionado a esse item, parâmetro $b$. Os parâmetros gerados estão em escala com métrica $(0,1)$.

$\mathrm{O}$ parâmetro $a$ mede o grau de discriminação do item. $\mathrm{Na}$ análise de satisfação, ele diferencia clientes com níveis distintos de satisfação em determinado ponto da escala. Este parâmetro representa o grau de inclinação da curva característica do item: quanto maior for o parâmetro, maior é a capacidade de diferenciar clientes com níveis distintos de satisfação.

Caso o item tenha o parâmetro $a$ muito baixo ele deve ser eliminando. Vargas (2007) restringe a eliminação a itens com a $<1$ enquanto que Alexandre et al. (2002) eliminam itens com a $<0,7$. Neste trabalho adotou-se a restrição do parâmetro a em 0,7 .

Observa-se, na Tabela 1, que nenhum item tem parâmetro inferior a 0,7 , ou seja, todos os itens apresentam bom grau de discriminação. Os itens com maior grau de discriminação, em ordem decrescente, foram os itens 17,31 e 16 e estão relacionados ao elemento atendimento. $\mathrm{O}$ conjunto de itens tem grau de discriminação médio 1,263 com desvio padrão de 0,353 .

O parâmetro $b$ fornece o grau de satisfação vinculado ao item na escala. Quanto maior o parâmetro $b$, menor a probabilidade de um cliente pouco satisfeito responder positivamente ao item. Assim, itens com $b$ baixo obtêm resposta positiva de uma quantidade maior de clientes. Segundo os resultados obtidos para o parâmetro $b$, tem-se os itens 1,25 e 2 relacionados aos maiores níveis de satisfação e os itens 9, 24 e 21 relacionados aos níveis mais baixos. É importante verificar a existência de itens relacionados a diferentes níveis de satisfação, indicando que o instrumento é capaz de estimar níveis de satisfação de clientes situados em pontos distintos da escala.

A partir do parâmetro $b$ de cada item é possível identificar o nível de satisfação que este item tem maior capacidade de avaliar. A Tabela 2 apresenta a classificação dos itens segundo o parâmetro $\mathrm{b} \mathrm{em}$, ordem decrescente.

Os produtos (rentabilidade e juros) e disponibilidade de estacionamento avaliam os clientes com maiores níveis de satisfação. Pode-se relacioná-los aos clientes 


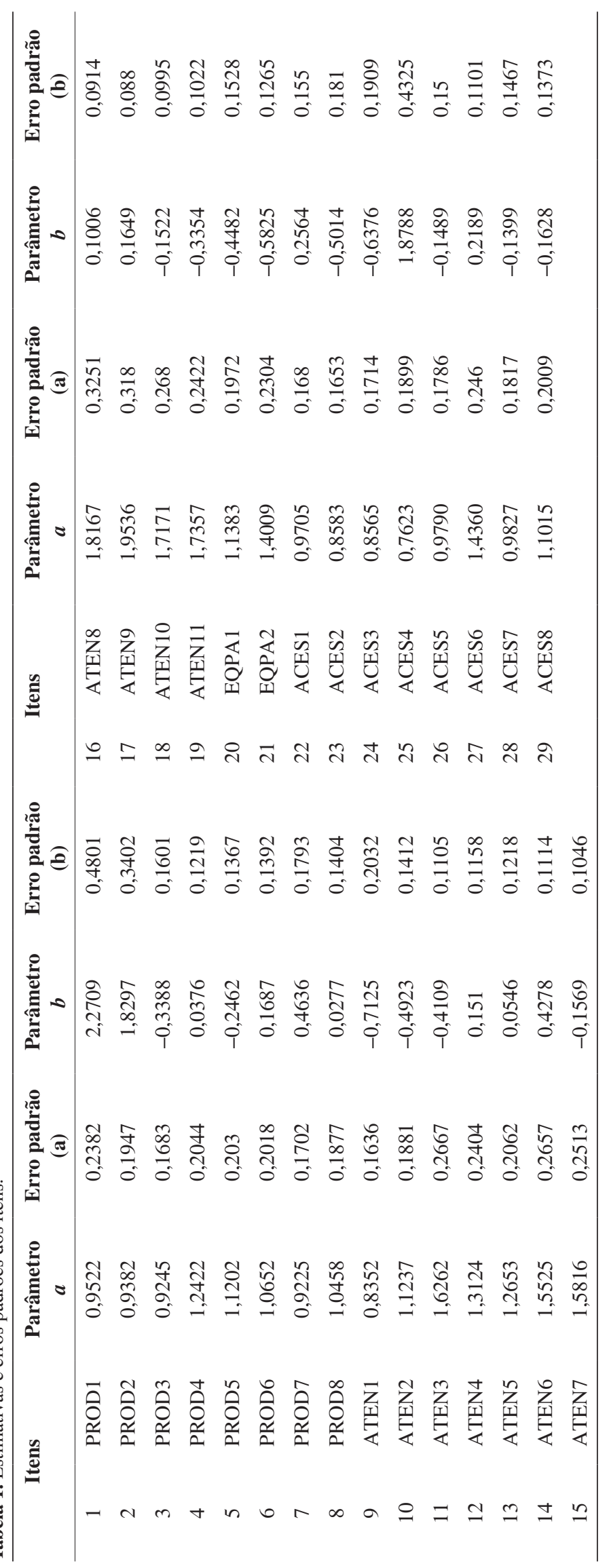


que atingem um nível mais elevado da amostra. Somente clientes com níveis de satisfação elevados têm alta probabilidade de resposta positiva a estes itens.

Itens relacionados ao atendimento, que avaliam o tempo gasto para resolver problemas, de resposta a reclamações, cumprimento de prazo. Ao acesso, que avaliam a segurança na agência. Aos produtos, relacionados à adequação e benefícios dos produtos, diferenciam clientes no nível médio da escala (nível 0). Aparência dos funcionários, equipamentos e sistemas e espaço físico avaliam clientes com menores níveis de satisfação.

Na Tabela 1, além dos parâmetros, são apresentados os erros padrões associados. É importante verificar a variação destes erros, e os casos com variação muito discrepante devem ser investigados. Observa-se, na Tabela 1, que os erros padrões relacionados às estimativas dos parâmetros dos itens $1,2 \mathrm{e}$ 25 destacam-se por causa dos valores superiores aos demais, o que poderia indicar inconsistência no processo de calibração. Realizou-se um teste excluindo-se esses três itens e verificou-se que os demais valores mantiveram-se estáveis. Por isso, decidiu-se manter estes itens na análise.

A capacidade dos itens avaliarem diferentes níveis de informação é representada pela curva de informação do teste. A Figura 3 apresenta a curva produzida a partir dos itens propostos. Considerando uma escala $(0,1)$, o instrumento concentra maior volume de informação entre os níveis de satisfação $(-1,50)$ e $(1,50)$. É significante a capacidade de avaliação do instrumento, pois a amostra utilizada apresenta nível de satisfação concentrado neste intervalo (Figura 3).

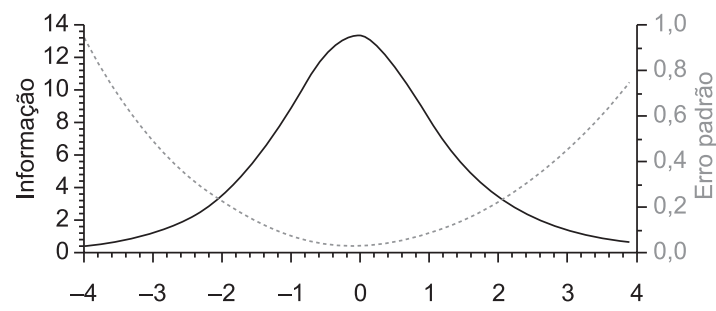

Figura 3. Curva de informação do teste.

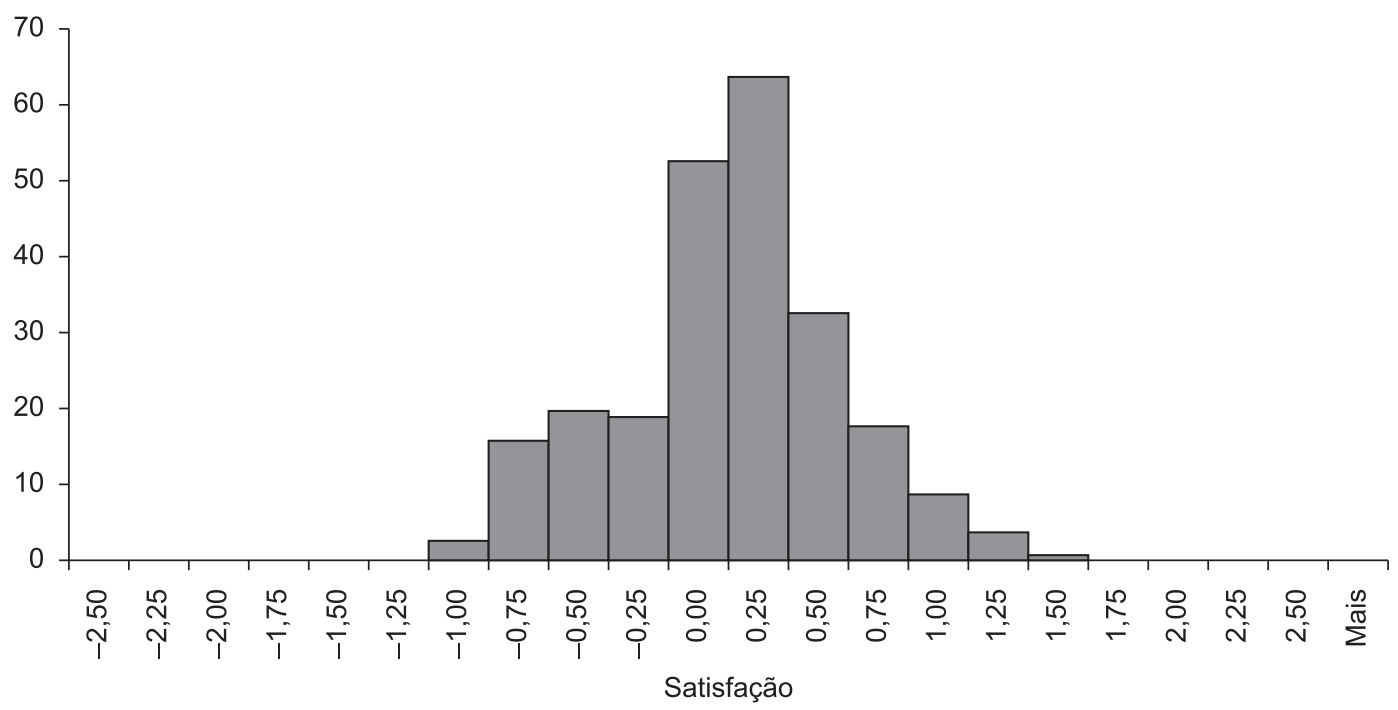

Figura 4. Distribuição da amostra conforme nível de satisfação.

Tabela 2. Classificação dos itens conforme nível de satisfação.

\begin{tabular}{rlcrccrcc}
\hline \multicolumn{1}{l}{ Item } & Parâmetro b & \multicolumn{2}{c}{ Item } & Parâmetro b & \multicolumn{2}{c}{ Item } & Parâmetro b \\
\hline 1 & PROD1 & 2,2709 & 16 & ATEN8 & 0,1006 & 19 & ATEN11 & $-0,3354$ \\
25 & ACES4 & 1,8788 & 13 & ATEN5 & 0,0546 & 3 & PROD3 & $-0,3388$ \\
2 & PROD2 & 1,8297 & 4 & PROD4 & 0,0376 & 11 & ATEN3 & $-0,4109$ \\
7 & PROD7 & 0,4636 & 8 & PROD8 & 0,0277 & 20 & EQPA1 & $-0,4482$ \\
14 & ATEN6 & 0,4278 & 28 & ACES7 & $-0,1399$ & 10 & ATEN2 & $-0,4923$ \\
22 & ACES1 & 0,2564 & 26 & ACES5 & $-0,1489$ & 23 & ACES2 & $-0,5014$ \\
27 & ACES6 & 0,2189 & 18 & ATEN10 & $-0,1522$ & 21 & EQPA2 & $-0,5825$ \\
6 & PROD6 & 0,1687 & 15 & ATEN7 & $-0,1569$ & 24 & ACES3 & $-0,6376$ \\
17 & ATEN9 & 0,1649 & 29 & ACES8 & $-0,1628$ & 9 & ATEN1 & $-0,7125$ \\
12 & ATEN4 & 0,151 & 5 & PROD5 & $-0,2462$ & & & \\
\hline
\end{tabular}


A partir dos parâmetros dos itens, é possível estimar o nível de satisfação dos clientes da amostra, Figura 4. A partir da distribuição dos níveis de satisfação, pode-se afirmar que $100 \%$ dos clientes atingem, no mínimo, o nível de satisfação -1 . À medida que se eleva o nível de satisfação, $75,83 \%$ dos clientes atingem o nível 0 . No nível 0,5 da escala, tem-se $27,08 \%$. Apenas $0,42 \%$ dos clientes atingem o nível 1,5.

Destaca-se que o modelo da TRI utilizado neste trabalho assume que clientes com um determinado nível de satisfação também devem estar satisfeitos com itens relacionados aos níveis inferiores. A amostra está concentrada na área da escala em que há maior informação, segundo a Curva de informação do teste, o que leva a concluir que o instrumento é adequado à amostra avaliada.

Portanto, o instrumento proposto e a escala criada com o uso da TRI mostraram-se adequadas para avaliar a satisfação dos clientes de bancos. Foi possível definir os parâmetros dos itens, identificar o nível de satisfação da amostra e relacionar os itens aos diferentes níveis de satisfação da escala apresentada.

\section{Conclusões}

Por meio de uma pesquisa quantitativa, este trabalho teve como objetivo elaborar um instrumento de medida para avaliar a satisfação de clientes de bancos que utilizam a Teoria da Resposta ao Item.

A partir de uma pesquisa bibliográfica, foram identificados 29 itens que abrangem características da atividade bancária relacionados ao atendimento, produtos, acesso e equipamentos e sistemas. Esses itens foram respondidos por 240 clientes, de forma a avaliar o banco com o qual possuíam maior relacionamento.

O teste dos itens, utilizando a Teoria da Resposta ao Item, permite considerar que os itens são adequados para medir a satisfação de clientes de bancos comerciais.

Os itens que avaliam a rentabilidade, os custos dos produtos e a disponibilidade de estacionamento apresentam um parâmetro $b$ maior. Portanto, representam características da atividade que discriminam clientes com nível de satisfação mais alto, ao passo que itens relacionados à aparência dos funcionários, equipamentos e sistemas e espaço físico discriminam clientes com menores níveis de satisfação.

Considerando uma escala com métrica $(0,1)$, analisando a curva de Informação do Teste, verifica-se que o conjunto de itens proposto estima com precisão níveis de satisfação entre -1,5 e 1,5. A partir dos parâmetros dos itens, foram estimados os níveis de satisfação da amostra.

Comparando-se Curva de Informação do Teste com os níveis de satisfação estimados, verificou-se que os últimos estavam concentrados na área da escala em que o teste possui maior volume de informação (maior precisão). Assim, concluiu-se que o instrumento de medida proposto é adequado para mensurar a satisfação de clientes de bancos, de acordo com a Teoria da Resposta ao Item. Recomenda-se para estudos futuros a expansão do tamanho da amostra, permitindo assim, utilizar itens com respostas ordinais, por exemplo. Também se pode aplicar o conjunto de itens proposto para comparar o desempenho de unidades de atendimento de um banco, assim como fazer comparações entre os resultados de diversos bancos, identificando pontos fortes. A aplicação de outros modelos da TRI para análise dos dados, tais como o multivariado ou o modelo de desdobramento, também pode ser explorada em futuras aplicações.

\section{Referências}

ALEXANDRE, J. W. C. et al. Uma proposta de análise de um construto para a medição dos fatores críticos da gestão pela qualidade por intermédio da teoria da resposta ao item. Gestão e Produção, v. 9, n. 2, p. 129-141, 2002.

ANDRADE, D. F.; TAVARES, H. R.; VALLE, R. C. Teoria da resposta ao item: conceitos e aplicações. São Paulo: Associação Brasileira de Estatística - ABE, 2000.

BAKER, F. B. The basics of item response theory. 2th ed. Washington: Eric Publications, 2001.

BANCO CENTRAL DO BRASIL. Disponível em: <www. bcb.gov.br>. Acesso em: 18 mar. 2010.

BATENSON, J. E. G.; HOFFMAN, K. D. Marketing de serviços. 4. ed. Porto Alegre: Bookman, 2001.

BEERLI, A.; MARTÍN, J. D.; QUINTANA, A. A model of customer loyaltiy in the retail banking market. European Journal of Marketing, v. 38, n. 1-2, p. 253-275, 2004. http://dx.doi.org/10.1108/03090560410511221

BERRY, L. L.; ZEITHAML, V. A.; PRASURAMA, A. Five imperatives for improving service quality. In: LOVELOCK, C. H. (Org). Managing services: marketing, operations, and human resources. 2 th ed. Englewood Cliffs: Prentice Hall, 1992.

CARUANA, A. Service Loyalty: the effects of service quality and the mediating role of customer satisfaction. European Journal of Marketing, v. 36. n. 7-8, p. 811-829, 2002. http://dx.doi.org/10.1108/03090560210430818

CHOWDHARY, N.; PRAKASH, M. Prioritizing service quality dimensions. Managing Service Quality, v. 17, n. 5, p. 493-509, 2007. http://dx.doi. org/10.1108/09604520710817325

CRONIN J. J.; TAYLOR, S. A. Measuring service quality: a reexamination and extension. Journal of Marketing, p. 55- 68, 1992. http://dx.doi.org/10.2307/1252296

FEDERAÇÃO BRASILEIRA DOS BANCOS FEBRABAN. O setor bancário em números - CIAB Febraban 2009. Disponível em: <http://www.febraban. org.br>. Acesso em: fev. 2009.

GIL, A. C. Métodos e técnicas de pesquisa social. São Paulo: Atlas, 1991.

GOSLING, M.; GONÇALVES, C. A. Relacionamento em bancos comerciais: Adaptação de escalas. Revista Eletrônica de Administração, v. 9, n. 4, p. 86-103, 2003. 
GOUNARIS, S.; ESTATHAKOPOULOS, V. Behavioral responses to customer satisfaction: an empirical study. European journal of marketing, v. 24, n. 5-6, p. 687-707, 2001.

HANSEMARK, O. C.; ALBINSSON, M. Customer satisfaction and the retention: the experiences of individual employees. Managing Service Quality, v. 14, n. 1, p. 40-57, 2004. http://dx.doi. org/10.1108/09604520410513668

HORTA, E. M. F. Avaliação da satisfação de médias empresas no relacionamento bancário. 2002. 198 f. Dissertação (Mestrado em Engenharia de Produção)-Universidade Federal de Santa Catarina, Florianópolis, 2002.

JAMAL, A.; NASER, K. Customer satisfaction and retail banking: na assessment of some of the key antecedents of customer satisfaction in the retail banking. International Journal of Bank Marketing, v. 20, n. 4, p. 146-160, 2002. http://dx.doi.org/10.1108/02652320210432936

JOHNSTON, R. Identifying the critical determinants of service quality in retail banking: importance and effect. International journal of bank marketing, v. 14, n. 4, p. 111-116, 1997. http://dx.doi. org/10.1108/02652329710189366

JURAN, J. M. A qualidade desde o projeto: os novos passos para o planejamento da qualidade em produtos e serviços. 3. ed. São Paulo: Pioneira, 1997.

LADHARI, R. Alternative measures of service quality: a review. Managing Service Quality,v. 18, n. 1, p. 66-85, 2006.

MANRAI, L. A.; MANRAI, A. K. A field study of customers' switching behavior for bank services. Journal of Retaling and Consumer Services, v. 14. p. 208-215, 2007. http://dx.doi.org/10.1016/j. jretconser.2006.09.005

MIGUEL, A. M. Determinantes da satisfação de clientes com os serviços bancários em Belo Horizonte. 2002. 179 f. Dissertação (Mestrado em Engenharia de Produção)-Universidade Federal de Santa Catarina, Florianópolis, 2002.

MIHELIS, G. et al. Customer satsifaction measurement in the private bank setor. European Journal of Operational Research, v. 130, p. 347-360, 2001. http://dx.doi. org/10.1016/S0377-2217(00)00036-9

MOUTINHO, L.; SMITH, A. Modelling bank customer satisfaction through mediation of attitudes towards human and automated banking. International Journal of Bank Marketing, v. 18, n. 3, p. 124-134, 2000. http:// dx.doi.org/10.1108/02652320010339699

NEWMAN, Karin. Interrogating SERVQUAL: a critical assessment of service quality mensurament in high street retail bank. International Journal of Bank Marketing. v. 19, n. 3. 2001, p. 126-139.

NUNES, C. H. S. S.; PRIMI, R. Impacto do tamanho da amostra na calibração de itens e estimativa de escores por teoria de reposta ao item. Avaliação Psicológica, v. 4, n. 2, p. 141-153, 2005.
OLORUNNIWO, F.; HSU, M. K.; UDO, G. F. Service quality, customer satisfaction, and behavioral intentions in the service factory. Journal of Services Marketing, v. 20, n. 1, p. 59-72, 2006. http://dx.doi. org/10.1108/08876040610646581

PALADINI, E. P. Gestão da qualidade: Teoria e Prática. 3. ed. São Paulo: Atlas, 2006.

PARASURAMAN, A.; ZEITHAML, V.; BERRY, L. SERVQUAL: a multiple-item scale for measuring consumer perception of service quality. Journal of Retaling, v. 64, n. 1, p. 12-40, 1988.

PATRÍCIO, L.; FISK, R.; CUNHA, J. F. Improving satisfaction with bank service offerings: measuring the contribution of each delivery channel. Managing Service Quality, v. 13, n. 6, p. 471-482, 2003. http:// dx.doi.org/10.1108/09604520310506531

PRIMI, R. Avanços na interpretação de escalas com a aplicação da teoria de resposta ao item. Avaliação psicológica, v. 3, n. 1, p. 53-58, 2004.

REISE, S.; AINSWORTH, A. T.; HAVILAND, M. G. Item response theory: fundamentals, applications, and promise in psychological research. Current Directions in Psychological Science, v. 14, n. 2, p. 95-101, 2005.

SOARES, T. M. Utilização da teoria da resposta ao item na produção de indicadores sócio econômicos. Revista Pesquisa Operacional, v. 25, n. 1, p. 83-112, 2005.

SURESHCHANDAR, G. S.; RAJENDRAN, C.; ANANTHARAMAN, R. N. The relationship between service quality and customer satisfaction - a factor specific approach. Journal os Services Marketing, v. 16, n. 4, p. 363-379, 2002.

UTTARO, T.; LEHMAN, A. Graded response modeling of quality of life interview. Evaluation and Program Planning, v. 22, p. 41-52, 1999. http://dx.doi.org/10.1016/ S0149-7189(98)00039-1

VANNIARAJAN, T.; ANBAZHAGAN, B. Servperf analysis in retail banking. International Marketing Conference on Marketing \& Society, v. 8, n. 10, p. 102-116, 2007.

VARGAS, V. C. C. Medida padronizada para avaliação de intangíveis organizacionais por meio da teoria da resposta ao item. 2007. Tese (Doutorado em Engenharia de Produção)-Universidade Federal de Santa Catarina, Florianópolis, 2007.

ZACHARIAS, M. L. B.; FIGUEIREDO, K. F.; ALMEIDA, V. M. C. Determinantes da satisfação dos clientes com serviços bancários. RAE - Eletrônica, v. 7, n. 2, 2008.

ZEITHAML, V. A.; BERRY, L. L.; PARASURAMAN, A. The Behavioral consequneces of service quality. Journal of Marketing, v. 60, p. 31-46, 1996. http:// dx.doi.org/10.2307/1251929

ZHOU, L. A dimension-specific analysis of performanceonly measurement of service quality and satisfaction in China's retail banking. Journal of Services Marketing, v. 18, n. 7, p. 534-546, 2004. http://dx.doi. org/10.1108/08876040410561866 